\title{
Coil placement after clipping: endovascular treatment of incompletely clipped cerebral aneurysms
}

\section{Report of two cases}

\section{Michael Forsting, M.D., Friedrich K. Albert, M.D., Olav Jansen, M.D., Rüdiger von Kummer, M.D., Alfred Aschoff, M.D., Stefan Kunze, M.D., and Klaus Sartor, M.D.}

Departments of Neuroradiology and Neurosurgergy, University Hospital, University of Heidelberg, Germany

In up to $4 \%$ of patients whose aneurysms are microsurgically clipped, there is an expected or unexpected aneurysm residuum. The authors describe two patients in whom surgical clipping did not result in complete obliteration of the aneurysm sac and in whom a second operation was not believed to be the solution to the problem. In both patients complete occlusion of the aneurysm residuum was achieved via an endovascular approach. Using the Guglielmi detachable coil system, it was possible to place two platinum coils selectively into the aneurysms. The endovascular approach may be a good treatment option for all patients in whom surgical clipping does not result in complete obliteration of the aneurysm sac and reoperation is contraindicated or unacceptable to the patient.

Key Words * intracranial aneurysm * failed surgery * aneurysm residuum * embolization * coil

The definitive treatment for cerebral aneurysms today is microsurgical clipping. However, in up to $4 \%$ of patients one finds on postoperative angiography an expected or unexpected aneurysm residuum due to incomplete clipping.[5,7,16] Incomplete obliteration of an aneurysm may result in dangerous enlargement of the residual sac and serious or fatal rebleeding months or years after surgery.[4-7,15] This is supported by theoretical calculations, which indicate that the difference in static blood pressure between the systemic pressure in the artery and that in the lumen of the incompletely clipped aneurysm is only 1 to $2 \mathrm{~mm} \mathrm{Hg}$.[8] Therefore, the risk of rupture secondary to blood pressure constraints is likely to remain significant.

Depending on the individual patient's anatomical situation, a second surgical attempt to achieve complete occlusion may be considered.[5,9] Feuerberg, et al.,[7] however, reported that up to 50\% of neurosurgeons believe that a second surgical approach would not improve the situation. Knowing that additional surgery may fail once more, the patient must accept partial treatment in the hope that the residual aneurysm will not again become dangerous.

The availability of navigable microcatheters and detachable microcoils now makes it possible to enter partially clipped aneurysms and occlude them. In this report we describe two patients in whom microsurgical clipping did not result in complete occlusion of the aneurysm sac and a second operation 
was not considered to be an option. In both patients endovascular treatment of the aneurysm residua was performed.

\section{CASE REPORT}

\section{Case 1}

This 36-year-old man presented with acute headache and subsequent deterioration into coma with a fixed and dilated left pupil and a weakly reactive right pupil (Hunt and Hess Grade V).

Neuroimaging Examination. An initial computerized tomography (CT) scan showed a large left-sided intracerebral temporal lobe hematoma that appeared to be induced by an aneurysm (Fig. 1 upper left).

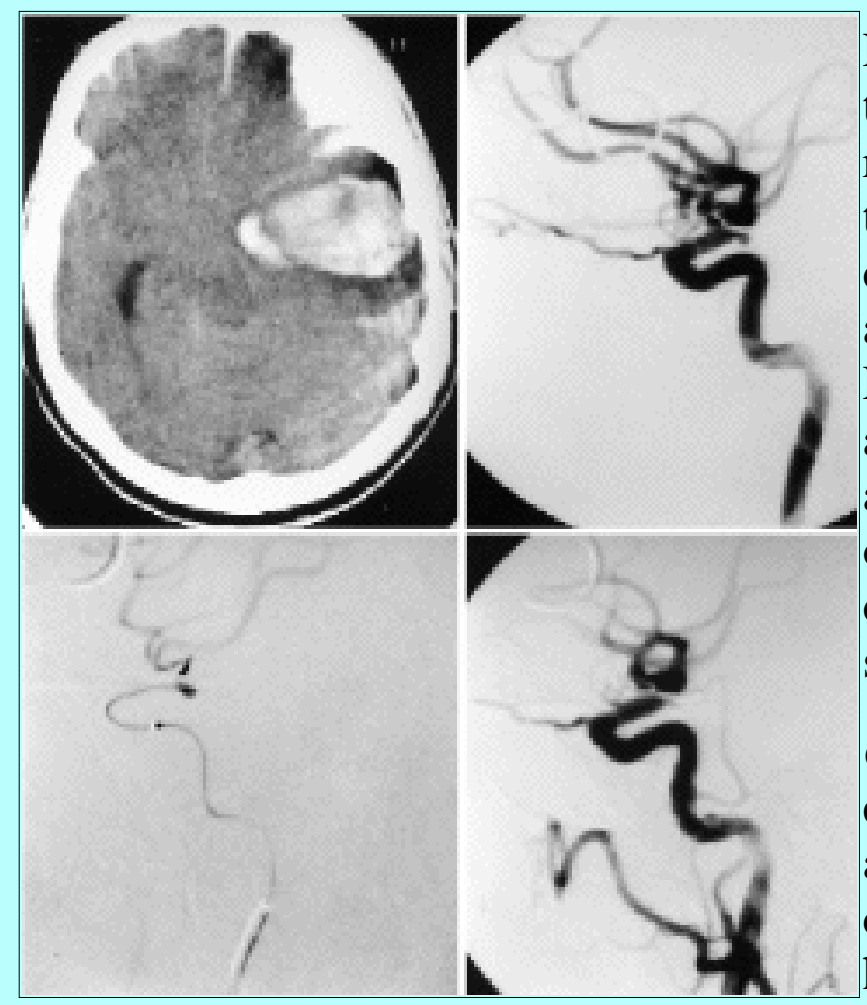

Fig. 1. Case 1. Upper Left: Axial computerized tomography scan obtained immediately after admission revealing a large intracerebral hematoma of the left temporal lobe. Upper Right: Postoperative left internal carotid angiogram, lateral view, displaying a small aneurysm residuum just proximal to the clip. Lower Left: Superselective angiogram, lateral view, obtained after the tip of the microcatheter was placed within the aneurysm residuum. Lower Right: Control angiogram of the left internal carotid artery obtained after detachment of two Guglielmi detachable coils, showing complete occlusion of the residual aneurysm.

Operation. Because of the poor clinical status of the patient, emergency surgery was performed without preoperative angiography. After evacuation of the hematoma, an internal carotid artery (ICA) aneurysm was found and clipped; however, the surgeon was uncertain about the degree of obliteration achieved. Severe brain swelling precluded a complete inspection of the aneurysm neck.

Postoperative Course. After gradual improvement of the patient in the intensive care unit, digital subtraction (DS) angiography revealed a small aneurysm residuum just proximal to the clip (Fig. 1 upper right). No other aneurysm was found. Two follow-up angiograms obtained within the next 10 months revealed absence of thrombosis within this small residuum, as well as a slight increase in size. During this time the patient had recovered more or less completely and was working again. Because repeat surgery was considered to be difficult and unlikely to produce a successful result, we decided to try an endovascular approach for complete obliteration.

Endovascular Therapy. One year after the patient's initial subarachnoid hemorrhage (SAH), endovascular treatment was performed while the patient was under general anesthesia and receiving heparin therapy to avoid thromboembolic complications. A Tracker No. 18 catheter was advanced into the aneurysm sac and two Guglielmi detachable coils (GDCs; Target Therapeutics, Fremont, CA) were placed selectively (Fig. 1 lower left).

Posttreatment Course. Control angiography showed complete occlusion of the aneurysm residuum with 
dense packing of the coils (Fig. 1 lower right). Three days later the patient was discharged in excellent clinical condition and immediately returned to work.

\section{Case 2}

This 37-year-old woman presented with acute headache followed by a loss of consciousness that lasted approximately 15 minutes.

Examination and History. At the time of admission her neurological condition was classified as a Hunt and Hess Grade II to III. Her initial CT scan showed marked SAH with a preponderance of blood in the right sylvian fissure and a right temporal lobe hematoma.

Ten years earlier the patient had suffered an SAH that was treated by clipping an aneurysm of the right posterior communicating artery. During that operation the aneurysm sac was opened after placement of the clip. A postoperative angiogram was not obtained at that time. At her current admission, angiography revealed an aneurysm just proximal to the clip (Fig. 2 upper left).

Fig. 2. Case 2. Upper Left: Right internal carotid angiogram, lateral view, obtained 10 years after clipping of an internal carotid artery (ICA) aneurysm, revealing a recurrent aneurysm of the ICA. Upper Right: Right internal carotid angiogram obtained after a second clip was placed (the 10-year-old clip remains in place). Note the aneurysm residuum between both clips. Lower Left and Right: Right internal carotid angiograms, lateral views, obtained immediately (lower left) and 9 months (lower right) after coiling of the aneurysm residuum. Note the complete occlusion of the aneurysm residuum.

Operation. During surgery a second clip was placed. The older, 10-year-old clip could not be removed because scar tissue tethered the aneurysm to the dural surface.

Intraoperatively, the surgeon had the impression of residual

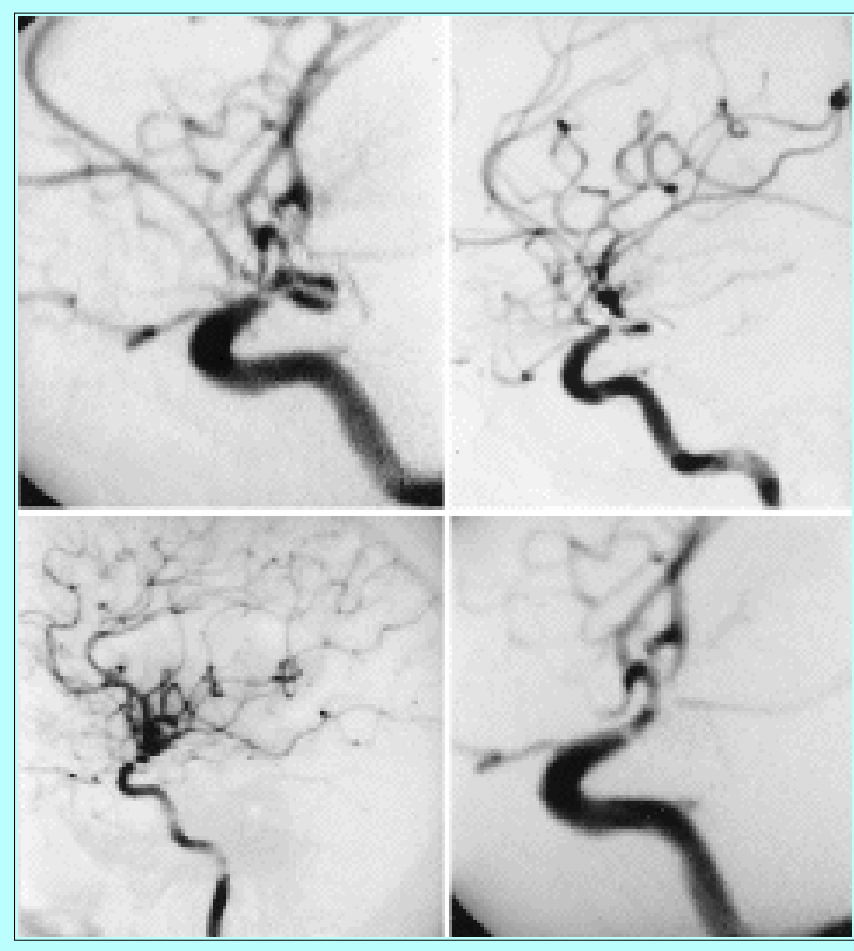
aneurysm just between the clips but its presence could not be avoided.

Endovascular Therapy. Digital subtraction angiography performed 10 days postsurgery confirmed a small aneurysm residuum between the two clips (Fig. 2 upper right). Subsequently endovascular treatment was performed in the same setting. Again, in this case it was not difficult to advance a Tracker No. 18 microcatheter into the aneurysm sac and to place two GDCs selectively.

Posttreatment Course. The initial control angiogram and two follow-up angiograms obtained within the next 9 months confirmed complete obliteration of the aneurysm sac (Fig. 2 lower left and right). In the meantime, the patient underwent surgery for another, albeit asymptomatic, aneurysm of the contralateral ICA. The patient was discharged in excellent neurological condition.

\section{DISCUSSION}

Recurrent signs of hemorrhage, routine postoperative angiography, repeat surgical exposure, and autopsy 
have documented many incidences of inadequate clipping in which part of the aneurysm remains at risk of clip slippage. There are only a few studies in the literature that deal with this problem, but the rate of incomplete obliteration of the aneurysm sac after microneurosurgical clipping has been estimated at approximately $4 \% .[4,5,7,18,19]$ This finding raises questions about the role of angiography after aneurysm clipping and about the management of residual aneurysm.

The prevailing opinion is that only postoperative angiography can document the results of operative obliteration of intracranial aneurysms.[14,16] Even opening an aneurysm sac after clipping does not safeguard against aneurysm residuum. Our second patient presented with recurrent SAH 10 years after her aneurysm had been clipped and its sac opened. This is similar to the findings of Drake and colleagues,[5] who reported an average time of 10.5 years between failed surgery and rebleeding.

Additionally, imperfect clip placement or a slipped clip may remain unrecognized until a postoperative angiogram is obtained. However, in many institutions (including ours), the standard practice is to perform postoperative angiography mainly in patients in whom difficulty was encountered at surgery.[14] The reason for this obvious diagnostic gap may be twofold: first, there is a small risk of angiographic complications, and second, it is sometimes difficult to know what to do with aneurysm residua. With regard to the first point, Macdonald and coworkers[16] found that postoperative angiography was safe with no evidence of neurological complication. They observed unclipped aneurysms in $4 \%$, unexpected residual portions of aneurysms in $4 \%$, and unexpected major vessel occlusions in $12 \%$ of their patients.[16]

With regard to the second reason, for a long time the prognostic significance of postoperative residual aneurysm was not sufficiently documented. One reason for this is that a traditional time frame (5 years) may not be sufficient to define the efficacy and the natural history of residual aneurysm.[9] Drake and coworkers [5] have concluded from their investigations that aneurysm residua are dangerous and that, if possible, a second operation should be performed to achieve a complete occlusion. However, Sato and Suzuki[19] indicated that the chances of spontaneous obliteration of the aneurysm residuum are good and the risk of a repeat rupture is not high. In a more recent report, Feuerberg and colleagues[7] found that the incidence of rebleeding from an aneurysm residuum is $3.7 \%$ and that the risk of rupture is up to $0.8 \%$ per year. They believe these results warrant reoperation of the residual aneurysm at least in young patients.[7] However, these data must be related to the risks of reoperation given by Drake and coworkers: an operative morbidity rate of 7\% and a mortality rate of 5\%.[5] In addition, there are considerable numbers of patients with residual aneurysm sacs in whom reoperation is considered impossible due to their anatomical conditions. In particular, scarring near the neck or fundus of the aneurysm may inhibit the ability to secure the aneurysm completely.[9] Knowing that reoperation will no doubt fail once more, these patients must accept partial treatment in the hope that the sac will not again become dangerous.

More recently, intraoperative angiography has proved to be an aid in evaluating the outcome of clipping in aneurysm surgery.[1,17] Intraoperative DS angiography, however, has been found to have a $4 \%$ false-negative rate, with failure to identify residual aneurysm filling.[1] Factors responsible for these failures include inadequate angiographic projections, inability to provide an image parallel to the clip blades, and the lower spatial resolution of portable DS angiography systems. Nevertheless, if available, intraoperative DS angiography can further improve the already excellent results of aneurysm surgery.

Advances in endovascular techniques have opened new avenues in the treatment of intracranial 
aneurysms.[10] In particular, use of GDCs has been successful and effective for treating some saccular aneurysms (both ruptured and unruptured).[2,11-13] Under ideal conditions, GDC treatment can be performed immediately after review of the diagnostic angiogram, adding only 1 to 2 hours to the diagnostic angiographic procedure.

All of the published series are small and their follow-up reviews are short. The reported total occlusion rate for GDC-treated aneurysms is approximately $80 \%$.[2] Therefore, this method is currently not an alternative to conventional microsurgery for most readily accessible aneurysms in the anterior portion of the circle of Willis. However, as far as we know, it is easier to achieve a complete occlusion with the GDC technique in aneurysms with a small neck. Therefore, a postsurgical situation in which there is a partially clipped and thus narrowed neck may be ideal to achieve complete occlusion using an endovascular approach. In 1994 Fraser and coworkers[8] first described the use of endovascular coils in incompletely clipped aneurysms in two patients; in one of these patients they also used the GDC system.[8] In all four patients described in the literature (Fraser's and ours) there were no problems with entering the aneurysm: in all patients a Tracker No. 18 catheter bypassed the clipped branches and coil deposition was possible.

In our patients complete occlusion of the postoperative aneurysm residuum was achieved via an endovascular technique. When such a complete aneurysm occlusion is accomplished with platinum coils there have been no subsequent reports of rebleeding.[2,3,11] Additionally, Graves, et al.,[11] reported that all of the completely occluded aneurysms in his series remained $100 \%$ occluded during follow up. No aneurysm that was initially completely occluded developed an aneurysm residuum.[11]

If GDC treatment should result in an incomplete obliteration of the aneurysm sac or a reopening due to coil compression, a second endovascular approach is possible. Moreover, treatment with GDCs does not preclude surgical clipping. Graves, et al.,[11] reported on two patients in whom surgical clipping of incompletely coiled aneurysms was performed without problems. We had a similar experience with a patient in whom coil placement did not result in a complete occlusion and surgical clipping was performed. Therefore, if the endovascular approach is deemed the method of choice, even in patients in whom a second surgical attempt seems to be possible, a failure of the endovascular treatment attempt does not preclude a second surgical approach, although in single cases the "coil ball" might pose a problem during placement of the clip.

The combination of a partial surgical clipping with narrowing of the aneurysm neck and subsequent endovascular treatment of aneurysm residuum may be a new therapy for those aneurysms that cannot be treated by either modality alone. If only partial clipping of a broad-based aneurysm can be performed (due to heavy calcification of the vessel wall or extension of the aneurysm wall into inaccessible locations) with surgical creation of a neck, then subsequent coil placement has a higher chance of success.

In conclusion, an endovascular approach using the GDC system may be a good therapeutic alternative for those patients in whom microsurgical clipping results in incomplete occlusion of the aneurysm and in whom reoperation is considered contraindicated or is unacceptable to the patient. In our patients no problems were encountered advancing a microcatheter into the aneurysm residuum and it may even be advantageous if the neck is already narrowed by a first clip. In two patients we achieved complete occlusion of aneurysms. However, there will remain some patients who cannot be treated by GDC embolization because the partially clipped aneurysm neck may still be too wide to retain coils or may be 


\section{References}

1. Barrow DL, Boyer KL, Joseph GJ: Intraoperative angiography in the management of neurovascular disorders. Neurosurgery 30:153-159, 1992

2. Byrne JV, Molyneux AJ, Brennan RP, et al: Embolisation of recently ruptured intracranial aneurysms. J Neurol Neurosurg Psychiatry 59:616-620, 1995

3. Casasco AE, Aymard A, Gobin YP, et al: Selective endovascular treatment of 71 intracranial aneurysms with platinum coils. J Neurosurg 79:3-10, 1993

4. Drake CG, Allcock JM: Postoperative angiography and the "slipped" clip. J Neurosurg 39:683-689, 1973

5. Drake CG, Friedman AH, Peerless SJ: Failed aneurysm surgery. Reoperation in 115 cases. J Neurosurg 61:848-856, 1984

6. Drake CG, Vanderlinden RG: The late consequences of incomplete surgical treatment of cerebral aneurysms. J Neurosurg 27:226-238, 1967

7. Feuerberg I, Lindquist C, Lindqvist M, et al: Natural history of postoperative aneurysm rests. J Neurosurg 66:30-34, 1987

8. Fraser KW, Halbach VV, Teitelbaum GP, et al: Endovascular platinum coil embolization of incompletely surgically clipped cerebral aneurysms. Surg Neurol 41:4-8, 1994

9. Giannotta SL, Litofsky NS: Reoperative management of intracranial aneurysms. J Neurosurg 83:387-393, 1995

10. Graves VB, Partington CR, Rüfenacht DA, et al: Treatment of carotid artery aneurysms with platinum coils and balloons: an experimental study in dogs. AJNR 11:249-252, 1990

11. Graves VB, Strother CM, Duff TA, et al: Early treatment of ruptured aneurysms with Guglielmi detachable coils: effect on subsequent bleeding. Neurosurgery 37:640-648, 1995

12. Guglielmi G, Viñuela F, Duckwiler G, et al: Endovascular treatment of posterior circulation aneurysms by electrothrombosis using electrically detachable coils. J Neurosurg 77:515-524, 1992

13. Guglielmi G, Viñuela F, Sepetka I, et al: Electrothrombosis of saccular aneurysms via endovascular approach. Part 1: Electrochemical basis, technique, and experimental results. J Neurosurg 75:1-7, 1991

14. Kassell NF: Angiography after aneurysm surgery. J Neurosurg 80:953, 1994 (Letter)

15. Lin T, Fox AJ, Drake CG: Regrowth of aneurysm sacs from residual neck following aneurysm clipping. J Neurosurg 70:556-560, 1989

16. Macdonald RL, Wallace MC, Kestle JRW: Role of angiography following aneurysm surgery. J Neurosurg 79:826-832, 1993

17. Martin NA, Bentson J, Viñuela F, et al: Intraoperative digital subtraction angiography and the 
surgical treatment of intracranial aneurysms and vascular malformations. J Neurosurg 73:526-533, 1990

18. Paterson A: Direct surgery in the treatment of posterior communicating aneurysms. Lancet 2:808-811, 1968

19. Sato S, Suzuki J: Prognosis in cases of intracranial aneurysm after incomplete direct operations. Acta Neurochir 24:245-252, 1971

Manuscript received February 21, 1996.

Accepted in final form May 15, 1996.

Address reprint requests to: Michael Forsting, M.D., Department of Neuroradiology, Im Neuenheimer Feld 400, D-69120 Heidelberg, Germany. 